\section{(6) OPEN ACCESS}

\title{
Hidradenitis suppurativa: a common and burdensome, yet under-recognised, inflammatory skin disease
}

\author{
Deirdre Nathalie Dufour, ${ }^{1}$ Lennart Emtestam, ${ }^{2}$ Gregor B Jemec ${ }^{1}$
}

${ }^{1}$ Department of Dermatology, Health Sciences Faculty, Roskilde Hospital, University of Copenhagen, Copenhagen, Denmark

${ }^{2}$ Department of Dermatology, Karolinska University Hospital and Karolinska Institutet, Stockholm, Sweden

\section{Correspondence to}

Professor L Emtestam, Department of Dermatology, Karolinska University Hospital and Karolinska Institutet, Stockholm SE-141 86, Sweden; Lennart.Emtestam@ki.se

Received 26 March 2013 Revised 14 November 2013 Accepted 13 January 2014 Published Online First 24 February 2014

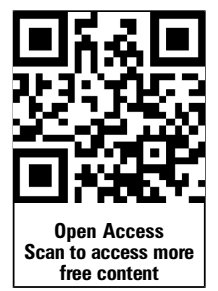

\footnotetext{
To cite: Dufour DN, Emtestam L, Jemec GB. Postgrad Med J 2014:90:216-221.
}

\begin{abstract}
Hidradenitis suppurativa (HS) is a chronic, relapsing, inflammatory skin condition that typically occurs after puberty. The primary clinical presentation is painful inflamed nodules or boils in the apocrine gland-bearing regions (armpits, genital area, groin, breasts and buttocks/anus) that progress to abscesses, sinus tracts and scarring. Severity is typically described according to three Hurley categories, with most patients having mild or moderate disease. Estimated prevalence is $1-4 \%$ worldwide and HS is three times more common in women than men. Patients' disease burden includes intense pain, work disability and overall poor quality of life. Although the clinical signs of the disease can often be hidden by clothing, active HS is associated with a malodorous discharge that contributes to the disabling social stigma. Risk factors include smoking and obesity. Comorbidities include inflammatory bowel disease and spondyloarthropathies. The presentation of the disease is distinct, yet HS is not well-recognised except in dermatology clinics.
\end{abstract}

\section{INTRODUCTION}

Hidradenitis suppurativa (HS), sometimes referred to as acne inversa, is a common, chronic, relapsing, inflammatory skin condition that greatly affects patients' quality of life. Its prevalence of $1-4 \% 0^{12}$ is similar to that of psoriasis. ${ }^{3}$ The patient with HS presents with inflammation of hair follicles in the apocrine gland-bearing regions (armpits, genital area, groin, inframammary region, perianal region and buttocks) that initially manifests as painful nodules or boils and progresses to abscesses, sinus tracts and scarring. ${ }^{4}$ The disease is often seen by non-dermatologists and clinical experience suggests that it is under-recognised. The overall disease burden is disproportionate to the estimated prevalence, and patients with HS not seen by dermatologists may not get timely and appropriate treatment if the condition is not identified, yet the clinical presentation is distinct and a reliable diagnosis can be made based on simple questions. ${ }^{5}$

We aimed to summarise the understanding of risk factors (eg, smoking, obesity, sex differences) and possible comorbidities (eg, inflammatory bowel diseases, spondyloarthropathies, epithelial tumours, pyoderma gangrenosum) and to discuss the problems of greatest relevance for patients with $\mathrm{HS}$ (eg, extreme pain, malodorous discharge, social stigma/psychosocial impact/isolation, work disability) so that awareness of this disease might be heightened in primary care physicians or general practitioners, who may be the first providers to see patients with this disease. Topics such as aetiology, pathogenesis, therapeutic options and clinical management clearly are important, but are beyond the scope of this review; however, these topics have been reviewed in other recent publications. ${ }^{6-11}$

\section{PREVALENCE, PATIENT CHARACTERISTICS AND SEX DIFFERENCES}

Before a study in a young Danish population undergoing screening for sexually transmitted diseases $(\mathrm{N}=507)$, in which Jemec et $a l^{1}$ found a point prevalence of $4.1 \%$, the prevalence of HS had not been estimated systematically (figure 1). The 1-year prevalence in this unselected sample of the general population in Denmark $(\mathrm{N}=599)$ was $1 \% .^{1}$ More recently, Revuz et $a l^{2}$ reported a prevalence of $1 \%$ in a representative sample of the French population $(\mathrm{N}=6887)$; however, the prevalence increased to $1.4 \%$ in the sample aged $<55$ years. In comparison, the prevalence of psoriasis is about $1.5-2 \%$ in industrialised nations. ${ }^{3}$

The average age at onset of HS is the early 20s, after puberty and the disease typically is active during the third and fourth decades of life. ${ }^{12} \mathrm{HS}$ is more common in women, with a female:male ratio of 3.3:1. ${ }^{12}{ }^{13}$ Although significant sex differences have been noted in patients with HS, plasma androgen concentrations (testosterone, sex hormone binding globulin and dehydroepiandrosterone sulfate) suggest that hyperandrogenism in women does not play a role in the development of HS after controlling for age, body mass index (BMI) and hirsutism. ${ }^{14}$ The armpit and inguinofemoral regions are the most common locations for HS lesions in both sexes. ${ }^{13}$ Frontal lesions (groin/thigh and breast) tend to be more common in women, whereas lesions in the buttocks, perineal/perianal regions and atypical areas (ears, chest) tend to be more common in men. ${ }^{13}$

\section{RISK FACTORS: SMOKING, OBESITY AND FAMILY HISTORY}

Several studies suggest an association between HS and smoking, obesity, family history and other patient factors (figure 1). In a matched case-control study in Germany, the odds of having HS were 9.4 times greater in current smokers $(89 \%)$ than in nonsmokers or ex-smokers (11\%). ${ }^{15}$ In both populationbased and clinic-based case-control studies, Revuz et $a l^{2}$ demonstrated an association with current smoking, but not prior smoking, and an increased prevalence of HS in a representative French sample. Another study found smoking to be associated with increased severity of $\mathrm{HS}^{16}$; non-smokers had 


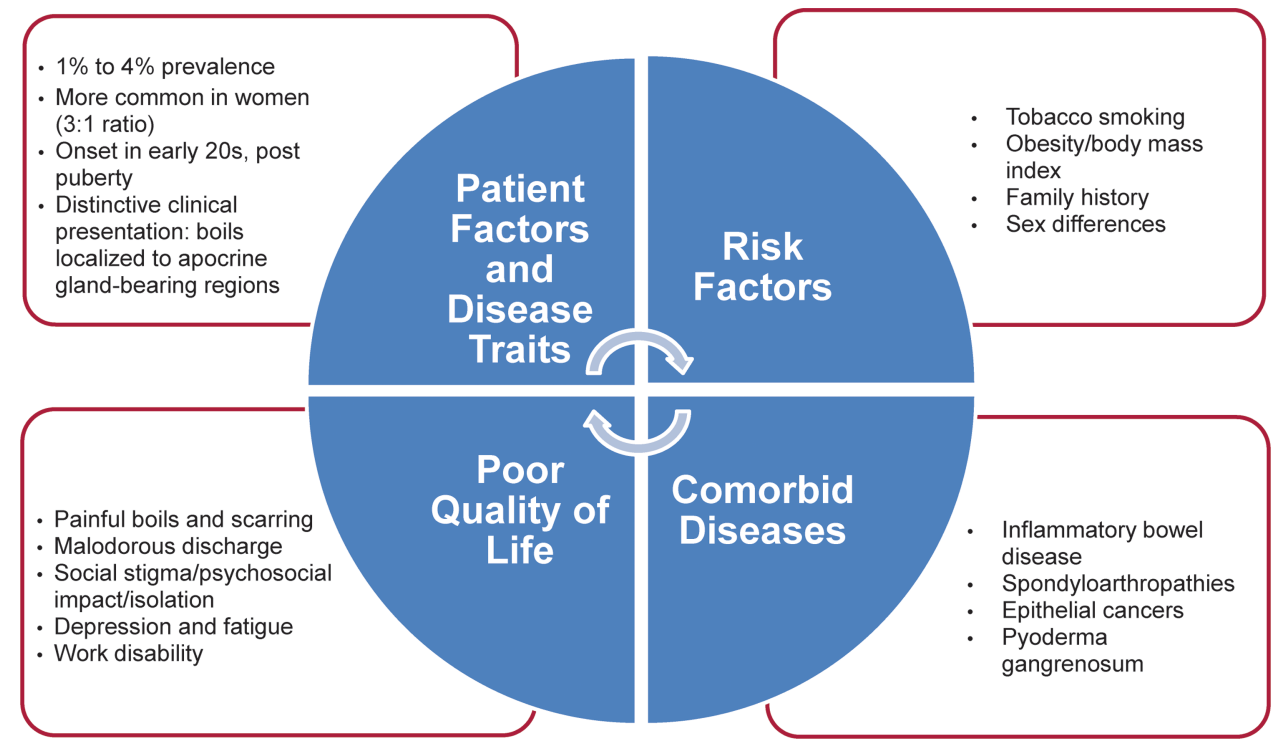

Figure 1 Disease burden in the patient with hidradenitis suppurativa (HS).

significantly lower median severity scores than smokers, and former smokers had intermediate scores. In contrast, univariate analysis of data from 302 French patients at a single referral centre failed to find an association with smoking and severity of HS. ${ }^{13} \mathrm{It}$ is not known whether smoking cessation affects the disease course of HS.

Studies also suggest that BMI is associated with HS prevalence and severity. For each unit increase in BMI, the risk for HS increased by 1.12 in a clinic-based sample. ${ }^{2}$ Likewise, Canoui-Poitrine et $a l^{13}$ found a strong association between BMI and the severity of HS in a referral centre population; for each unit increase in BMI, the HS score increased by 0.84 units. When divided into BMI categories of normal, overweight and obese, obese patients had more severe HS than overweight patients, and overweight patients had more severe HS than normal weight patients. ${ }^{16}$ A recent study in which patients with HS had a higher prevalence of metabolic syndrome than controls suggests that metabolic abnormalities may be a contributing factor in the development of HS, particularly in younger patients. ${ }^{17}$

Family history studies have found a positive family history of HS in about one in three patients and an autosomal dominant inheritance pattern has been suggested. ${ }^{18} 19$ No association has been found with HLA-B or HLA-DR types, ${ }^{20}$ and an initial report suggesting involvement of chromosome $1 \mathrm{p} 21.1-1 \mathrm{q} 25.3^{21}$ was not confirmed in two large, multigeneration pedigrees (96 individuals, 25 affected). ${ }^{19}$ CARD15 polymorphisms were also excluded in a pilot study and a case series. ${ }^{22}$ An association with the gamma secretase gene was found in one study of cases with an atypical and particularly severe phenotype of the disease, ${ }^{24}$ consequently extrapolation to the general population of patients with HS is difficult.

Other studies have found somewhat mixed associations between HS and smoking, body weight and other risk factors. In a study of 54 Polish patients ( 28 women, 26 men) who qualified for HS surgery, Matusiak et $a l^{25}$ corroborated smoking as a potentially causative factor in HS but found no, or equivocal, associations with BMI, diabetes mellitus, hyperandrogenism and profuse sweating. In addition, shaving the affected areas before HS onset and positive family history were associated with earlier disease onset. ${ }^{25}$ Jemec et $a l^{26}$ found no association with cosmetics, oral contraceptives or BMI among 68 consecutive patients treated by dermatologists in Denmark.

\section{CLINICAL PRESENTATION AND DISEASE SEVERITY}

Inflammation of the apocrine gland-bearing regions causing painful boils is the hallmark presentation of HS, the severity of which is determined by the degree to which the lesions progress to abscesses, sinus tracts and scarring. Severity is typically described according to the three Hurley categories (table 1). ${ }^{27}$ Most patients have grades I (mild) or II (moderate) HS, with grade III (severe) disease reported in $4-22 \%$ of patients in recent studies. ${ }^{13} 1625$ A typical patient with moderate HS might be a 26-year-old woman with multiple, widely separated, painful, recurrent abscesses as well as sinus tracts and cicatrisation under her breasts or armpits (figure 2A,B); she might have a family history of symptoms consistent with HS and she might be a smoker with BMI in the obese range. Sinus tracts and cicatrisation would be absent in mild HS, whereas involvement would be diffuse with multiple interconnected tracts and abscesses in severe HS (figure 2C). Increased BMI, atypical locations, history of severe acne and absence of a family history of HS have been identified as independent factors associated with increased disease severity. ${ }^{13}$ In addition, men are more likely

Table 1 Hurley severity for hidradenitis suppurativa (HS)

\begin{tabular}{|c|c|c|}
\hline $\begin{array}{l}\text { Degree of } \\
\text { involvement }\end{array}$ & Definition $^{27}$ & $\begin{array}{l}\text { Reported range } \\
\text { of patients* } \\
\text { affected }^{131625}(\%)\end{array}$ \\
\hline Grade I & $\begin{array}{l}\text { Abscess formation, single or multiple, } \\
\text { without sinus tracts and cicatrisation }\end{array}$ & $7-68$ \\
\hline Grade II & $\begin{array}{l}\text { Recurrent abscesses with sinus tracts } \\
\text { and cicatrisation; single or multiple } \\
\text { widely separated lesions }\end{array}$ & $28-83$ \\
\hline Grade III & $\begin{array}{l}\text { Diffuse or almost diffuse involvement, or } \\
\text { multiple interconnected tracts and } \\
\text { abscess across entire area }\end{array}$ & $4-22$ \\
\hline \multicolumn{3}{|c|}{$\begin{array}{l}\text { *Populations: Canoui-Poitrine et al }{ }^{13} \text { included } 302 \text { consecutive HS referrals in France; } \\
\text { Sartorius et } a l^{16} \text { included } 251 \text { consecutive referrals to a clinic with interest in HS in } \\
\text { Sweden ( } 115 \text { with HS); and Matusiak et al }{ }^{25} \text { included } 54 \text { Polish patients with HS who } \\
\text { qualified for surgical intervention. }\end{array}$} \\
\hline
\end{tabular}


Figure 2 Typical clinical presentation of active hidradenitis suppurativa (HS). (A) Moderate Hurley grade II. (B) Severe Hurley grade II. (C) Hurley grade III. (a)

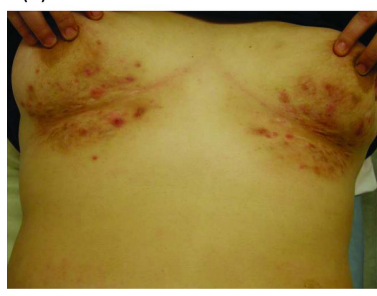

(b)

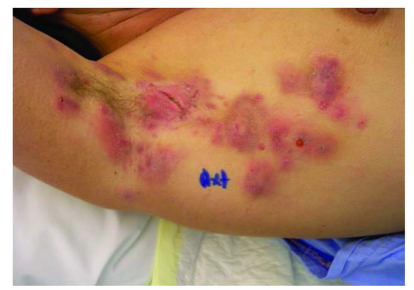

(c)

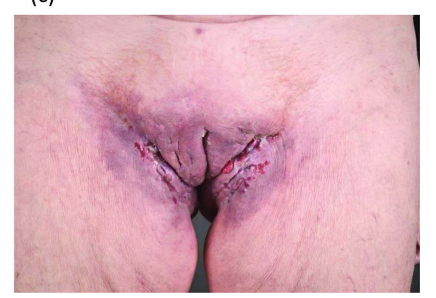

than women to develop severe HS, ${ }^{13}{ }^{25}$ and associations between smoking and obesity and more severe disease have been reported. ${ }^{16}$

\section{COMORBID CONDITIONS: INFLAMMATORY BOWEL DISEASE, SPONDYLOARTHROPATHY, TUMOURS AND PYODERMA GANGRENOSUM}

Case reports have described a number of possible comorbid diseases; however, the validity of the observations is limited by their numbers, and further studies are needed (figure 1). Some of the associations described, such as anaemia and amyloidosis, are clearly linked to disease severity and, therefore, qualify better as complications than as comorbid diseases. ${ }^{28} 29$ Several possible comorbidities have been systematically investigated, including autoinflammatory diseases (eg, inflammatory bowel disease and spondyloarthropathy), epithelial tumours and pyoderma gangrenosum.

For autoinflammatory diseases, one review found inflammatory bowel disease and spondyloarthropathies to be the most frequently reported comorbid conditions in patients with HS (82 and 59 cases, respectively, reported in the literature). ${ }^{30}$ In particular, it has been suggested that HS occurs often in patients with inflammatory bowel disease, raising the possibility of shared pathogenesis. ${ }^{31}$ Painful boils in the axillae and/or groin in patients with inflammatory bowel disease have been reported in $17 \%$ of patients with Crohn's disease and $14 \%$ of patients with ulcerative colitis in the Netherlands. ${ }^{31}$ In addition, the serendipitous observation that tumour necrosis factor $\alpha$ antibodies used in a patient with Crohn's disease also had a beneficial effect on the patient's HS has led to the development of new treatments for HS. ${ }^{32}$

An increase of epithelial and non-melanoma skin cancers has been reported in patients with HS. ${ }^{25} 3334$ Standardised incidence ratios in a Swedish population indicated that the risk for any cancer was $50 \%$ greater for patients with HS than for matched controls, with significantly increased risk for nonmelanoma skin cancer, buccal cancer and liver cancer. ${ }^{33}$ For squamous cell carcinoma, Lavogiez $e t a l^{34}$ found a prevalence of $4.6 \%$ in a sample of 217 cases and confirmed its predominance in men with potential involvement of human papillomavirus.

Although the association is rare, it has been speculated that pyoderma gangrenosum and HS share a common aetiology that may involve cytokine dysregulation. A retrospective medical chart review identified 11 cases of patients with HS and pyoderma gangrenosum, which is an uncommon inflammatory skin condition associated with large, painful ulcers, most often on the legs. ${ }^{35}$ In about half of the cases, pyoderma gangrenosum and HS lesions affected the same areas; however, this overlap of lesions was reported less often among 20 other cases identified in the literature.

\section{IMPACT ON QUALITY OF LIFE AND ABILITY TO WORK}

The physical symptoms of HS-namely, nodules or boils that progress to abscesses, sinus tracts and scarring, make it an extremely painful condition (figure 1). The pain associated with
HS can be intense and chronic and is reported by patients as the most significant factor contributing to impaired quality of life. ${ }^{36} 37$ On an 11-point scale (numeric rating scale-11), in which 0 represents no pain and 10 represents the worst imaginable or extreme pain, patients with HS typically rated their pain in the range 4-10 and described it as hot, burning, pressure, stretching, cutting, sharp, taut, splitting, gnawing, pressing sore, throbbing and aching. ${ }^{36}$ The average visual analogue scale pain score was 4.2 in 61 patients in a French population with HS. ${ }^{37}$ Soreness was the most common problem related to HS cited by both men and women in one study, followed by discharge and appearance for women. ${ }^{26}$

Although the clinical signs of the disease can often be hidden by clothing, the active condition is associated with malodorous discharge that stains clothing; thus, HS is accompanied by embarrassment, disabling social stigma, low self-worth and impact on interpersonal relationships. ${ }^{16} 263839$ Mean Dermatology Life Quality Index (DLQI) score in a group of 251 Swedish patients with HS was 10 (range 0-30; median 9), indicating substantial disease-specific impairment of quality of life. ${ }^{16}$ Patients scored items relating to soreness and pain, clothing and embarrassment/ self-consciousness as the most disabling; DLQI scores were not influenced by smoking or BMI status. ${ }^{16}$ Further, patients with HS had significantly worse self-reported quality of life than the general Danish population, with soreness, restriction of movement and social situations being the most problematic factors. ${ }^{26}$

Quality-of-life impairment in patients with HS exceeds that of other skin diseases that generally are perceived to have a high burden and substantial disability. ${ }^{37-39}$ The mean DLQI score was 8.9 in the first survey of patients with HS, which was numerically greater than scores in other studies of patients with alopecia (8.3), acne (7.5), mild to moderate psoriasis (7.0), Hailey-Hailey disease (6.1), Darier's disease (5.9), vascular anomalies of the face (5.6) and atopic dermatitis (5.5). ${ }^{37}$ Compared with patients with neurofibromatosis type 1 in another study, patients with HS had significantly worse disease-specific quality of life with regard to emotions, symptoms and function (Skindex-France). ${ }^{38}$ Similarly, patients with HS had worse self-perceptions and mood, more physical discomfort and greater impairment in daily activities and social functioning (VQ-Dermato) than patients with chronic urticaria, psoriasis and/or atopic dermatitis. ${ }^{38}$ In addition, patients with HS had poorer disease-specific quality of life and higher depression scores (as measured by the DLQI and Major Depression Inventory, respectively) than patients diagnosed with other dermatological diseases; these outcomes correlated with the severity of HS. ${ }^{39}$ Moreover, general health as measured by Short Form 36 Health Survey scores was also significantly worse for patients with HS than that for patients with neurofibromatosis and normal controls for seven of the eight dimensions (role-physical was numerically but not significantly worse for patients with HS). ${ }^{38}$

HS affects self-worth because of the ugly, malodorous physical symptoms and the lack of control of the disease. Consequently, patients often isolate themselves as a coping mechanism and are depressed. Using patient interviews $(\mathrm{N}=12)$ 
and qualitative methods, Esmann and Jemec ${ }^{40}$ identified several factors that contribute to the social stigma and psychosocial impact of HS. With respect to interpersonal relationships, patients reported the importance of being in a partnership and expressed interest in interacting with other patients with HS. Among a sample of 54 Polish patients with HS, ${ }^{41} 21 \%$ could be considered clinically depressed according to the Beck Depression Inventory-Short Form (scores $\geq 10$ ); clinically significant fatigue was identified in $40 \%$ of patients (Functional Assessment of Chronic Illness Therapy-Fatigue scores $>30$ ). Hurley disease severity categories were predictive of the extent of psychosocial impact, and scores on all questionnaires reflected increasing impairment with increasing disease activity; anogenital location was consistently associated with the worst outcomes. In general, patients with HS had greater rates of depression than patients with other skin diseases, and overall quality of life was as poor as serious medical conditions such as cancer, lung diseases and cardiovascular diseases. ${ }^{41}$

Onset and peak disease activity of HS occurs during the patient's productive years. Consequently, HS can affect a patient's ability to work. In a survey conducted in the UK, patients with HS reported a median of two boils a month, each with average duration of 6.9 days. $^{12}$ This duration equals a potential 14 days/month of active disease, and $62 \%$ of patients also reported at least one permanent tender lump. ${ }^{12}$ Patients with HS were absent from work for an average of 2.7 days per patientyear because of their disease (range 0-30 days). ${ }^{26}$ Among 30 working patients, $58 \%$ reported missing work because of their HS; such absences occurred 1-10 times a year and lasted for 396 days. $^{42}$

\section{SUMMARY}

HS is a relatively common and extremely burdensome inflammatory skin condition that is not well-recognised outside of

\section{Main messages}

- Hidradenitis suppurativa (HS) is a chronic, relapsing, inflammatory skin disease with a prevalence similar to that of psoriasis.

- The clinical presentation is distinctive: painful boils/ abscesses/scarring preferentially including the armpits, genitals, groin, inframammary region, perianal region and buttocks.

- Risk factors for HS include smoking and obesity; it is three times more common in women than men but often is more severe in men.

- HS is associated with extreme pain and malodorous discharge, which can lead to psychological stress, social stigma and work disability.

- HS has been reported to co-occur with inflammatory bowel diseases, spondyloarthropathies, epithelial cancers and squamous cell carcinoma and pyoderma gangrenosum.

- Patients' quality of life is poorer than that of patients with many other dermatological conditions and serious medical conditions. dermatology clinics; yet, the clinical presentation is distinct and awareness of common risk factors, possible comorbid conditions and patient-reported quality of life can enable a reliable diagnosis. With a prevalence of $1-4 \%$, HS is not a rare disease. It occurs most frequently in post-pubescent women, and sex differences have been identified. Risk factors for HS include smoking and obesity, which may also be associated with more severe disease. About one-third of patients report a family history of HS. HS has been reported to co-occur with several comorbid conditions-most notably, inflammatory bowel disease. HS is associated with a stigmatising malodorous discharge and patients with HS endure severe pain; these factors contribute to depression, work disability and overall poor quality of life. Heightened awareness of the important clinical and patient-reported features of HS among primary care physicians and general practitioners should lead to better care of patients with HS.

\section{Current research questions}

- How can studies of aetiology and pathogenesis, currently aimed at identifying underlying immunological mechanisms, help us to identify additional targets for treatment of hidradenitis suppurativa (HS)?

- What genes are linked to the HS phenotype?

- What systemic studies with traditional treatments, such as topical or systemic antibiotics, could offer insight into optimising treatment of HS?

- Tumour necrosis factor is raised in patients with HS and preliminary data suggest that biological agents may be effective for the worst cases of HS, but what is the overall benefit-risk and which is the appropriate target population for biological therapy?

\section{Key references}

- Canoui-Poitrine F, Revuz JE, Wolkenstein P, et al. Clinical characteristics of a series of 302 French patients with hidradenitis suppurativa, with an analysis of factors associated with disease severity. J Am Acad Dermatol 2009;61:51-7.

- Esmann S, Dufour DN, Jemec GB. Questionnaire-based diagnosis of hidradenitis suppurativa: specificity, sensitivity and positive predictive value of specific diagnostic questions. Br J Dermatol 2010;163:102-6.

- Esmann S, Jemec GB. Psychosocial impact of hidradenitis suppurativa: a qualitative study. Acta Derm Venereol 2011;91:328-32.

- Jemec GB. Clinical practice. Hidradenitis suppurativa. N Engl J Med 2012;366:158-64.

- Revuz JE, Canoui-Poitrine F, Wolkenstein P, et al. Prevalence and factors associated with hidradenitis suppurativa: results from two case-control studies. J Am Acad Dermatol 2008;59:596-601. 
Self assessment questions

Answer true $(T)$ or false $(F)$ for the below:

1. The following is a clinical or patient-reported feature of hidradenitis suppurativa (HS):

A. Pain, often intense, in the affected area

B. Inflamed nodules or boils in the apocrine gland-bearing regions (armpits, genital area, groin, breasts and buttocks/anus)

C. Malodorous discharge that can stain clothing

D. Raised, inflamed, red, scaly patches on the elbows knees, scalp and/or lower back

E. Psychosocial impact leading to quality-of-life impairment (social stigma, isolation, work disability)

2. The following condition is a risk factor for HS:

A. Smoking

B. Hyperandrogenism

C. Obesity

D. Female sex

E. Family history

3. The following condition has been reported to co-occur with HS:

A. Epithelial tumours/non-melanoma skin cancer

B. Inflammatory bowel disease

C. Psoriasis

D. Spondyloarthropathy

E. Pyoderma gangrenosum

4. The following characteristic has been associated with the clinical manifestations and/or epidemiology of HS:

A. Frontal lesions (groin/thigh and breast) more common in women

B. Greater frequency of severe HS in men

C. Lesions in the back of the body (buttocks and perianal/ perineal area) more common in men

D. Estimated prevalence (1-4\%), similar to that of psoriasis

E. Lesser quality-of-life impairment than with other dermatological conditions

5. This clinical presentation might best describe a typical patient with moderately severe (Hurley grade II) HS:

A. 73-year-old man with diffuse involvement, multiple interconnected cicatrisation and abscesses across entire area; no known family of HS; and non-smoker with normal body mass index (BMI)

B. 73-year-old woman with abscess on the front of her body but no sinus tracts or cicatrisation; family history of symptoms consistent with $\mathrm{HS}$; and smoker with BMI in the overweight range

C. 26-year-old woman with multiple, painful abscess on the front of her body but no sinus tracts or cicatrisation; family history of symptoms consistent with HS; and smoker with $\mathrm{BMI}$ in the obese range

D. 26-year-old woman with multiple, widely separated, painful, recurrent abscesses as well as sinus tracts and cicatrisation on the front of her body; family history of symptoms consistent with $\mathrm{HS}$; and smoker with $\mathrm{BMI}$ in the obese range

E. 26-year-old man with single, painful abscess on the back of his body but no sinus tracts or cicatrisation; family history of symptoms consistent with HS; and smoker with $\mathrm{BMI}$ in the obese range
Acknowledgements AbbVie provided suggestions for topic ideas and authors for consideration. Cathryn M Carter, MS, of Arbor Communications provided medical writing and editorial support to the authors in the development of this manuscript.

Contributors DND, LE, GBJ: specified the content of the manuscript, identified the relevant articles for inclusion, reviewed all drafts and revised critically for important intellectual content and approved the version to be submitted.

Funding AbbVie funded development of this manuscript.

Competing interests GBJ has been an investigator and speaker for AbbVie in the previous 3 years. DND has been an investigator for AbbVie in the previous 3 years.

Provenance and peer review Not commissioned; externally peer reviewed.

Open Access This is an Open Access article distributed in accordance with the Creative Commons Attribution Non Commercial (CC BY-NC 3.0) license, which permits others to distribute, remix, adapt, build upon this work non-commercially, and license their derivative works on different terms, provided the original work is properly cited and the use is non-commercial. See: http://creativecommons.org/ licenses/by-nc/3.0/

\section{REFERENCES}

1 Jemec $\mathrm{GB}$, Heidenheim M, Nielsen NH. The prevalence of hidradenitis suppurativa and its potential precursor lesions. J Am Acad Dermatol 1996;35(2 Pt 1):191-4.

2 Revuz JE, Canoui-Poitrine F, Wolkenstein $P$, et al. Prevalence and factors associated with hidradenitis suppurativa: results from two case-control studies. J Am Acad Dermatol 2008;59:596-601.

3 Nevitt GJ, Hutchinson PE. Psoriasis in the community: prevalence, severity and patients' beliefs and attitudes towards the disease. Br J Dermatol 1996;135:533-7.

4 Kurzen $\mathrm{H}$, Kurokawa I, Jemec $\mathrm{GB}$, et al. What causes hidradenitis suppurativa? Exp Dermatol 2008;17:455-6; discussion 457-72.

5 Esmann S, Dufour DN, Jemec GB. Questionnaire-based diagnosis of hidradenitis suppurativa: specificity, sensitivity and positive predictive value of specific diagnostic questions. Br J Dermatol 2010;163:102-6.

6 Jemec GB. The concept of 'smokers' boils' is suggestive of a new hypothesis on the pathogenesis of hidradenitis suppurativa. Dermatology 2011;222:196-7.

7 Nazary M, der Zee HV, Prens E, et al. Pathogenesis and pharmacotherapy of Hidradenitis suppurativa. Eur J Pharmacol 2011;672:1-8.

8 Yazdanyar S, Jemec GB. Hidradenitis suppurativa: a review of cause and treatment. Curr Opin Infect Dis 2011;24:118-23.

9 Rambhatla PV, Lim HW, Hamzavi I. A systematic review of treatments for hidradenitis suppurativa. Arch Dermatol 2012;148:439-46.

10 Jemec GB. Clinical practice. Hidradenitis suppurativa. N Engl J Med 2012;366:158-64

11 van der Zee HH, Laman JD, Boer J, et al. Hidradenitis suppurativa: viewpoint on clinical phenotyping, pathogenesis and novel treatments. Exp Dermatol 2012;21:735-9.

12 von der Werth JM, Williams HC. The natural history of hidradenitis suppurativa. J Eur Acad Dermatol Venereol 2000;14:389-92.

13 Canoui-Poitrine F, Revuz JE, Wolkenstein P, et al. Clinical characteristics of a series of 302 French patients with hidradenitis suppurativa, with an analysis of factors associated with disease severity. J Am Acad Dermatol 2009;61:51-7.

14 Barth JH, Layton AM, Cunliffe WJ. Endocrine factors in pre- and postmenopausal women with hidradenitis suppurativa. Br J Dermatol 1996;134:1057-9.

15 König A, Lehmann C, Rompel $R$, et al. Cigarette smoking as a triggering factor of hidradenitis suppurativa. Dermatology 1999;198:261-4.

16 Sartorius K, Emtestam L, Jemec GB, et al. Objective scoring of hidradenitis suppurativa reflecting the role of tobacco smoking and obesity. Br J Dermatol 2009;161:831-9.

17 Sabat R, Chanwangpong A, Schneider-Burrus S, et al. Increased prevalence of metabolic syndrome in patients with acne inversa. PLOS ONE 2012;7:e31810.

18 von der Werth JM, Williams HC, Raeburn JA. The clinical genetics of hidradenitis suppurativa revisited. Br J Dermatol 2000;142:947-53.

19 Al-Ali FM, Ratnamala U, Mehta TY, et al. Hidradenitis suppurativa (or acne inversa) with autosomal dominant inheritance is not linked to chromosome 1p21.1-1q25.3 region. Exp Dermatol 2010;19:851-3.

20 Lapins J, Olerup 0, Emtestam L. No human leukocyte antigen-A, -B or -DR association in Swedish patients with hidradenitis suppurativa. Acta Derm Venereol 2001:81:28-30.

21 Gao M, Wang PG, Cui Y, et al. Inversa acne (hidradenitis suppurativa): a case report and identification of the locus at chromosome 1p21.1-1q25.3. J Invest Dermatol 2006;126:1302-6.

22 Nassar D, Hugot JP, Wolkenstein P, et al. Lack of association between CARD15 gene polymorphisms and hidradenitis suppurativa: a pilot study. Dermatology 2007;215:359.

23 van Rappard DC, Mekkes JR. Hidradenitis suppurativa not associated with CARD15/ NOD2 mutation: a case series. Int J Dermatol 2014;53:e77-9.

24 Wang $B$, Yang W, Wen W, et al. Gamma-secretase gene mutations in familial acne inversa. Science 2010;330:1065. 
25 Matusiak L, Bieniek A, Szepietowski JC. Hidradenitis suppurativa and associated factors: still unsolved problems. J Am Acad Dermatol 2009;61:362-5.

26 Jemec GB, Heidenheim M, Nielsen NH. Hidradenitis suppurativa: characteristics and consequences. Clin Exp Dermatol 1996;21:419-23.

27 Hurley HJ. Axillary hyperhidrosis, apocrine bromhidrosis, hidradenitis suppurativa and familial benign pemphigus: surgical approach. In: Roenigk RK, Roenigk HH Jr, eds. Dermatologic surgery: principles and practice. 2nd ed. New York: Marcel Dekker, 1996:623-45.

28 Girouard SD, Falk RH, Rennke HG, et al. Hidradenitis suppurativa resulting in systemic amyloid A amyloidosis: a case report and review of the literature. Dermatol Online J 2012;18:2.

29 Tennant F Jr, Bergeron JR, Stone OJ, et al. Anemia associated with hidradenitis suppurativa. Arch Dermatol 1968;98:138-40.

30 Fimmel S, Zouboulis CC. Comorbidities of hidradenitis suppurativa (acne inversa). Dermatoendocrinol 2010;2:9-16.

31 van der Zee $\mathrm{HH}$, van der Woude CJ, Florencia EF, et al. Hidradenitis suppurativa and inflammatory bowel disease: are they associated? Results of a pilot study. $\mathrm{Br} \mathrm{J}$ Dermatol 2010;162:195-7.

32 Martínez F, Nos P, Benlloch S, et al. Hidradenitis suppurativa and Crohn's disease: response to treatment with infliximab. Inflamm Bowel Dis 2001;7:323-6.

33 Lapins J, Ye W, Nyrén 0, et al. Incidence of cancer among patients with hidradenitis suppurativa. Arch Dermatol 2001:137:730-4.

34 Lavogiez C, Delaporte E, Darras-Vercambre S, et al. Clinicopathological study of 13 cases of squamous cell carcinoma complicating hidradenitis suppurativa. Dermatology 2010;220:147-53.

35 Hsiao JL, Antaya RJ, Berger T, et al. Hidradenitis suppurativa and concomitant pyoderma gangrenosum: a case series and literature review. Arch Dermatol 2010;146:1265-70.
36 Smith HS, Chao JD, Teitelbaum J. Painful hidradenitis suppurativa. Clin J Pain 2010;26:435-44

37 von der Werth JM, Jemec GB. Morbidity in patients with hidradenitis suppurativa. Br J Dermatol 2001;144:809-13.

38 Wolkenstein P, Loundou A, Barrau K, et al. Quality of Life Group of the French Society of Dermatology. Quality of life impairment in hidradenitis suppurativa: a study of 61 cases. J Am Acad Dermatol 2007;56:621-3.

39 Onderdijk AJ, van der Zee HH, Esmann S, et al. Depression in patients with hidradenitis suppurativa. J Eur Acad Dermato/ Venereol 2013;27:473-8.

40 Esmann S, Jemec GB. Psychosocial impact of hidradenitis suppurativa: a qualitative study. Acta Derm Venereol 2011;91:328-32

41 Matusiak L, Bieniek A, Szepietowski JC. Psychophysical aspects of hidradenitis suppurativa. Acta Derm Venereol 2010;90:264-8.

42 Matusiak $飞$, Bieniek A, Szepietowski JC. Hidradenitis suppurativa markedly decreases quality of life and professional activity. J Am Acad Dermatol 2010;62:706-8.

\section{Answers}

1. (A) $\mathrm{T} ;$ (B) $\mathrm{T}$; (C) $\mathrm{T}$; (D) F; (E) T.

2. (A) $\mathrm{T} ;$ (B) $\mathrm{F}$; (C) $\mathrm{T}$; (D) $\mathrm{T}$; (E) $\mathrm{T}$.

3. (A) $\mathrm{T}$; (B) $\mathrm{T}$; (C) $\mathrm{F}$; (D) $\mathrm{T}$; (E) $\mathrm{T}$.

4. (A) $\mathrm{T} ;$ (B) $\mathrm{T} ;$ (C) $\mathrm{T}$; (D) $\mathrm{T}$; (E) $\mathrm{F}$.

5. (A) $\mathrm{F} ;(\mathrm{B}) \mathrm{F} ;(\mathrm{C}) \mathrm{F} ;(\mathrm{D}) \mathrm{T}$; (E) $\mathrm{F}$.

\section{Warwick University Short Course}

14-17 July 2014: 'Techniques and Applications of Molecular Biology: A Course for Medical Practitioners'. A four-day course for those in the medical profession wishing to improve their understanding of the principles and applications of genetic engineering techniques. Optional accreditation leads to a masters level Postgraduate Award.

Details: Dr Charlotte Moonan, School of Life Sciences, University of Warwick, Coventry CV4 7AL, UK; Tel: 0247652 3540; Email: Charlotte.Moonan@warwick.ac.uk; Website: http://www2.warwick.ac.uk/fac/sci/lifesci/study/shortcourses/molecularbiology). 